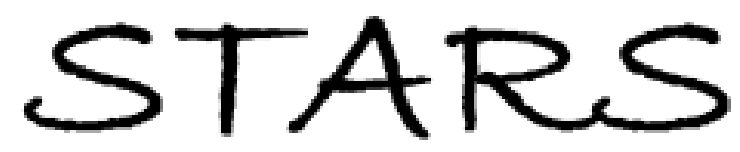

University of Central Florida

STARS

$1-1-2011$

\title{
Near-barrier quasielastic scattering as a sensitive tool to derive nuclear matter diffuseness
}

\author{
E. Crema \\ D. R. Otomar \\ R. F. Simões \\ A. Barioni \\ D. S. Monteiro
}

See next page for additional authors

Find similar works at: https://stars.library.ucf.edu/facultybib2010

University of Central Florida Libraries http://library.ucf.edu

This Article is brought to you for free and open access by the Faculty Bibliography at STARS. It has been accepted for inclusion in Faculty Bibliography 2010 s by an authorized administrator of STARS. For more information, please contactSTARS@ucf.edu.

\section{Recommended Citation}

Crema, E.; Otomar, D. R.; Simões, R. F.; Barioni, A.; Monteiro, D. S.; Ono, L. K.; Shorto, J. M. B.; Lubian, J.; and Gomes, P. R. S., "Near-barrier quasielastic scattering as a sensitive tool to derive nuclear matter diffuseness" (2011). Faculty Bibliography 2010s. 1216.

https://stars.library.ucf.edu/facultybib2010/1216

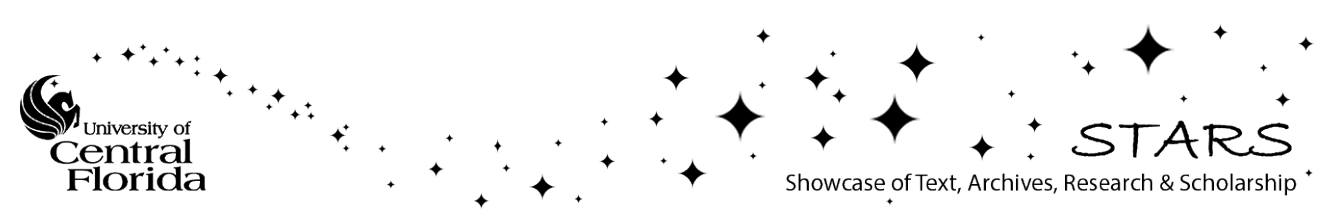




\section{Authors}

E. Crema, D. R. Otomar, R. F. Simões, A. Barioni, D. S. Monteiro, L. K. Ono, J. M. B. Shorto, J. Lubian, and P. R. S. Gomes 


\title{
Near-barrier quasielastic scattering as a sensitive tool to derive nuclear matter diffuseness
}

\author{
E. Crema,${ }^{1, *}$ D. R. Otomar, ${ }^{2}$ R. F. Simões,${ }^{1}$ A. Barioni, ${ }^{1}$ D. S. Monteiro, ${ }^{2}$ L. K. Ono, ${ }^{3}$ J. M. B. Shorto, ${ }^{4}$ \\ J. Lubian, ${ }^{2}$ and P. R. S. Gomes ${ }^{2}$ \\ ${ }^{1}$ Departamento de Física Nuclear, Instituto de Física da Universidade de São Paulo, Travessa R da Rua do Matão, 187, 05508-090, \\ São Paulo, São Paulo, Brazil \\ ${ }^{2}$ Instituto de Física, Universidade Federal Fluminense, Avenida Litorânea s/n, Gragoatá, Niterói, Rio de Janeiro, 24210-340, Brazil \\ ${ }^{3}$ Physics Department, University of Central Florida, 4000 Central Florida Boulevard, Orlando, Florida 32816, USA \\ ${ }^{4}$ Instituto de Pesquisas Energéticas e Nucleares, IPEN/CNEN, Avenida Lineu Prestes, 2242, 05508-000, São Paulo, São Paulo, Brazil
}

(Received 18 April 2011; revised manuscript received 31 May 2011; published 1 August 2011)

\begin{abstract}
Quasielastic excitation functions for the ${ }^{16,18} \mathrm{O}+{ }^{60} \mathrm{Ni}$ systems were measured at energies near and below the Coulomb barrier, at the backward angle $\theta_{\mathrm{LAB}}=161^{\circ}$. The corresponding quasielastic barrier distributions were derived. The data were compared with predictions from coupled channel calculations using a double-folding potential as a bare potential. For the ${ }^{16} \mathrm{O}$-induced scattering, good agreement was obtained for the barrier distribution by using the projectile default nuclear matter diffuseness obtained from the São Paulo potential systematic, that is, $0.56 \mathrm{fm}$. However, for the ${ }^{18} \mathrm{O}$-induced scattering, good agreement was obtained only when the projectile nuclear matter diffuseness was changed to $0.62 \mathrm{fm}$. Therefore, in this paper we show how near-barrier quasielastic scattering can be used as a sensitive tool to derive nuclear matter diffuseness.
\end{abstract}

DOI: 10.1103/PhysRevC.84.024601

PACS number(s): 25.70.Bc, 25.60.Pj, 25.70.Mn, 25.70.Jj

\section{INTRODUCTION}

Despite the progress that has been attained towards the production of light and heavy nuclei far from the stability valley, there is still a lack of experimental data of very basic nuclear properties of stable nuclei. One example is the size of a nucleus, because the average radius and the neutron skin diffuseness are quantities very difficult to measure [1]. While the electrical properties of a nucleus can be investigated directly with electrons or charged probes [2], the properties related with its neutron distribution cannot be easily accessed. Most of the available information on the nuclear matter radius and diffuseness comes from theoretical calculations [3]. Nuclear reaction theories have surmounted this problem by describing the nucleus-nucleus interaction as an optical potential with the Woods-Saxon shape, which has a diffuseness that should be related with the interacting nuclei diffuseness. However, the optical potential diffuseness and the nuclear matter diffuseness (NMD) of the interacting nuclei are different quantities. Some works have been dedicated to derive the interacting potential diffuseness for different systems from deep subbarrier quasielastic scattering data at backward angles, as, for example Refs. [4-9]. The derivation of the NMD from this kind of work is a difficult task, since the interacting potentials of Woods-Saxon form have several parameters and consequently have large ambiguities. In addition, the imaginary and real components of the potentials are dependent on each other, and related by a dispersion relation. In order to overcome these difficulties and to derive nuclear diffuseness of different isotopes, one should directly derive this quantity (NMD).

In a recent paper, we presented a method that, taking advantage of the strong influence of the interaction potential

*crema@if.usp.br on the quasielastic barrier distribution (QEBD), could derive the NMD of the ${ }^{17} \mathrm{O}$ nucleus [10]. Instead of using optical potentials, double-folding potentials were used as the real part of interaction potentials because these potentials are explicitly dependent on the charge and matter densities of each interacting nucleus. No imaginary potential was used at the surface interaction region because all relevant direct reaction channels were included in the coupling scheme. So, by varying the folding potential, and by fitting the QEBD with coupled channel calculations, we were able to derive the NMD of the ${ }^{17} \mathrm{O}$ nucleus, as $a=0.62 \mathrm{fm}$. The NMDs of the ${ }^{16} \mathrm{O}$ and ${ }^{64} \mathrm{Zn}$ were found to be $0.56 \mathrm{fm}$, a value that is the average matter diffuseness of a systematic over a large number of nuclei [3]. In the following, we will employ the same procedure to derive the NMD of the ${ }^{18} \mathrm{O}$ and ${ }^{60} \mathrm{Ni}$ nuclei.

For the tightly bound systems, at energies around the Coulomb barrier, quasielastic scattering (QES) is the sum of elastic scattering, inelastic excitations, and transfer channels, that is, all relevant reaction channels, except fusion. The QEBD is obtained from the first derivative, with respect to energy, of the ratio of the quasielastic differential cross section to the Rutherford differential cross section, $d\left(\sigma_{\text {qel }} / \sigma_{\text {Ruth }}\right) / d E$. It has been shown that near-barrier backward QES is a very useful tool to investigate coupling channel effects in systems involving heavy ions [11-20]. Besides, QEBD is a very interesting alternative tool to the derivation of fusion barrier distributions because fusion and backward QES are complementary processes that are mainly fed by the same lowest partial waves. Since these two reaction mechanisms at energies close to the Coulomb barrier are dominated by quantum tunneling processes, they are very sensitive to the potential's details. In addition, the derivative of the quasielastic excitation function that produces the QEBD acts like a filter that makes the barrier distribution also very sensitive to the interaction potential shape. These two properties of the QEBD at energies near the Coulomb barrier make our method a precise tool to measure the nuclear matter diffuseness (NMD). 
In the comparison between data and predictions from coupled channel calculations, a critical point is the choice of the bare potential to be used. Double-folding potentials are widely accepted as a reliable choice. Our group and others have successfully used the São Paulo potential (SPP) $[3,21]$ in the investigation of scattering, fusion, and other reaction mechanisms for a wide range of systems and energies, including fusion barrier distributions of weakly bound systems [22] and QEBD for tightly bound light systems [17,18,23,24]. The double-folding SPP uses the two-parameter-Fermi shape (2p-Fermi) for both the nuclear matter and charge densities, as in Eq. (1), where the average radius and diffuseness parameters are deduced from experimental scattering data and theoretical calculations for hundreds of systems [3]:

$$
\rho(r)=\frac{\rho_{o}}{1+\exp \left(\frac{r-R_{o}}{a}\right)} .
$$

The charge and nuclear matter radii follow very well systematic behaviors with little dispersion around the average values, which are calculated by $R_{\mathrm{CH}}=\left(1.76 Z^{1 / 3}-0.96\right) \mathrm{fm}$ and $R_{\mathrm{MATT}}=\left(1.31 A^{1 / 3}-0.84\right) \mathrm{fm}$, respectively [3]. However, this is not the case of diffuseness parameters, which, due to shell and structure effects, present a large dispersion around the average values, $0.53 \mathrm{fm}$ and $0.56 \mathrm{fm}$, for the charge and nuclear matter diffuseness, respectively [3]. Therefore it is important to have precise measurements of these diffuseness parameters.

In the present work we report the investigation of the nearbarrier quasielastic scattering of the ${ }^{16,18} \mathrm{O}+{ }^{60} \mathrm{Ni}$ systems. For the ${ }^{18} \mathrm{O}$-induced reactions, one expects that the transfer of neutrons plays an important role in the coupling scheme and acts as a doorway to fusion.

In Sec. II, we describe the experiments and results for the near-barrier QES measurements for the ${ }^{16,18} \mathrm{O}+{ }^{60} \mathrm{Ni}$ systems. In the same section, we compare the experimental barrier distributions for both systems. In Sec. III, we present the comparison of the experimental results with the predictions from coupled channel calculations (CCCs) for QES excitation function and QEBD for the ${ }^{16} \mathrm{O}+{ }^{60} \mathrm{Ni}$ system, using the NMD from the SPP systematic $(0.56 \mathrm{fm})$. The agreement for the barrier distribution is good. In Sec. IV, the same procedure is repeated for the ${ }^{18} \mathrm{O}+{ }^{60} \mathrm{Ni}$ system. However, for this system there is a strong disagreement between the predictions and the experimental results. We then let the NMD of the ${ }^{18} \mathrm{O}$ be a free parameter, and very good agreement is obtained for the ${ }^{18} \mathrm{O}$ diffuseness value of $0.62 \mathrm{fm}$. Finally, we present the conclusions.

\section{EXPERIMENTAL DETAILS AND RESULTS}

The experiments were performed at the Pelletron Laboratory of the University of São Paulo. Beams of ${ }^{16,18} \mathrm{O}$ were delivered at the bombarding energy range from 30.0 to $48.0 \mathrm{MeV}$. The beam energy was varied in steps of $0.5 \mathrm{MeV}$ at the barrier energy region, and $1.0 \mathrm{MeV}$ at energies well below and well above the barrier. The self-supporting ${ }^{60} \mathrm{Ni}$ targets were enriched to $99.5 \%$ and their thicknesses were $\sim 70 \mu \mathrm{g} / \mathrm{cm}^{2}$.

The detection system consisted of one $E-\Delta E$ telescope placed at $\theta_{\mathrm{LAB}}=161^{\circ}$, where the $\Delta E$ signal was provided by a gas proportional counter, and the residual energy $E$ was measured by a silicon surface barrier detector at its rear side. In addition, three surface barrier detectors were placed at forward angles $\left( \pm 30^{\circ}\right.$ and $\left.+45^{\circ}\right)$ relative to the beam direction, for normalization purposes and for monitoring the beam. The number of quasielastic events was higher than $10^{4}$, producing uncertainties in the quasielastic excitation function below $1 \%$. For the highest energies, where the cross sections were very small, the statistics were poorer than that and the uncertainties were $\sim 3 \%$. To reduce magnetic hysteresis effects, the main analyzing magnet was properly recycled before starting the measurements, and from then on the beam energy was decreased monotonically.

Figure 1 shows typical $E-\Delta E$ spectra, at $E_{\mathrm{LAB}}=41.0 \mathrm{MeV}$, for the ${ }^{16} \mathrm{O}+{ }^{60} \mathrm{Ni}$ [Fig. 1(a)] and ${ }^{18} \mathrm{O}+{ }^{60} \mathrm{Ni}$ [Fig. 1(b)]
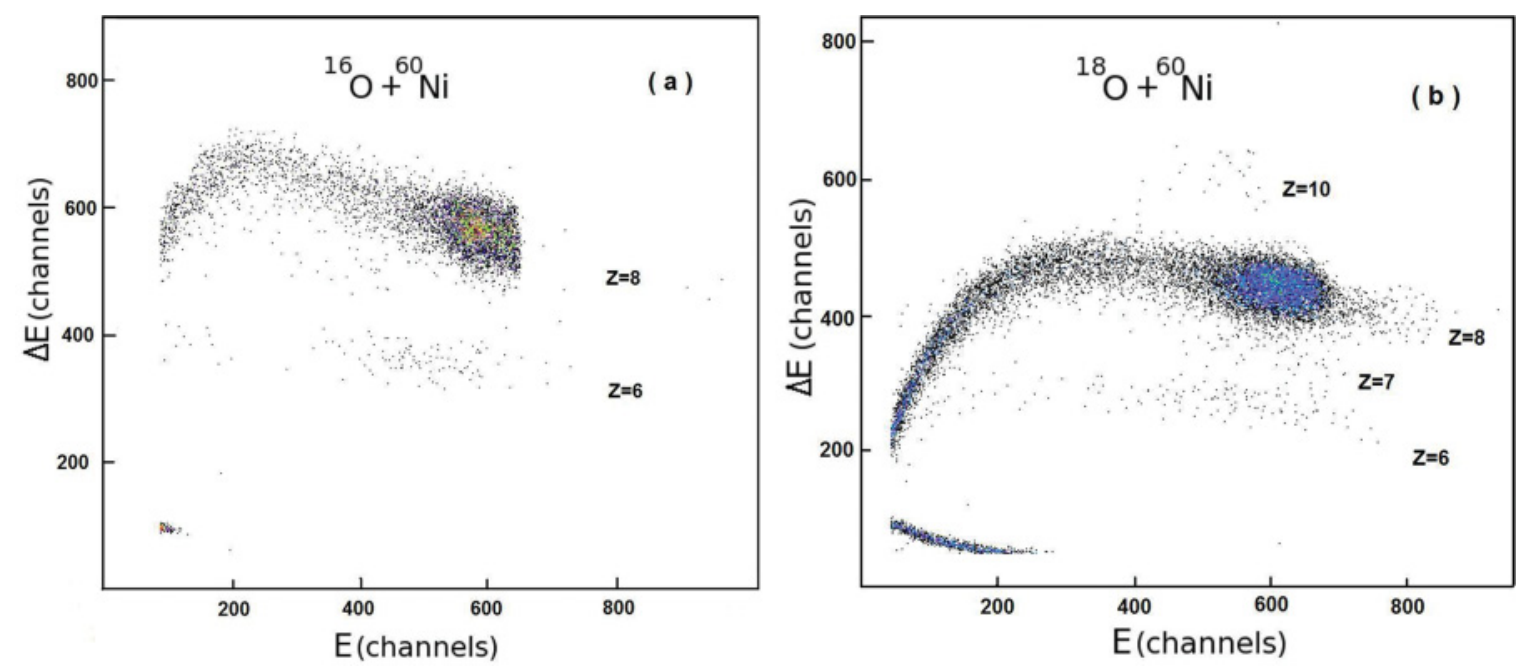

FIG. 1. (Color online) Typical $E-\Delta E$ spectra of (a) the ${ }^{16} \mathrm{O}+{ }^{60} \mathrm{Ni}$ and (b) ${ }^{18} \mathrm{O}+{ }^{60} \mathrm{Ni}$ systems, taken at $E_{\mathrm{LAB}}=41 \mathrm{MeV}$ and $\theta_{\mathrm{LAB}}=161^{\circ}$. 
systems, respectively. These spectra were taken at approximately the same experimental conditions (beam time, beam intensity, and target thickness) in order to allow a direct qualitative comparison between them. One can observe that the $Z$ resolution is good enough to identify the different reaction products.

In the ${ }^{16} \mathrm{O}+{ }^{60} \mathrm{Ni}$ system, the transfer mechanism is dominated by the $\alpha$-stripping process $[Z=6$ in Fig. 1(a)]. On the other hand, when the projectile is ${ }^{18} \mathrm{O}$, the presence of its two neutrons outside its double-magic ${ }^{16} \mathrm{O}$ core completely changes the reaction mechanism. This is easily observed in the spectrum shown in Fig. 1(b), where, besides the elastic and inelastic scattering $(Z=8)$, it is possible to distinguish several transfers of a few nucleons $(Z=6,7,9,10)$. However, the remarkable difference between the two spectra is the presence of neutron transfer processes with energies larger than the one for elastic scattering that is observed within the $Z=8$ events of the ${ }^{18} \mathrm{O}$ system. While in the ${ }^{16} \mathrm{O}+{ }^{60} \mathrm{Ni}$ system all neutron transfer channels have highly negative $Q_{\text {gg values, the }}{ }^{18} \mathrm{O}+$ ${ }^{60} \mathrm{Ni}$ system presents several transfer channels with positive, or near-zero, $Q_{\mathrm{gg}}$ values. Since the one- and two-neutron stripping processes have, respectively, $Q_{\mathrm{gg}}=-0.224 \mathrm{MeV}$ and $Q_{\mathrm{gg}}=+6.230 \mathrm{MeV}$, we may conclude that the events in Fig. 1(b) with $Z=8$ and energy above the one for elastic scattering may be mainly due to the two-neutron stripping process.

To improve the statistics required to derive barrier distributions, the acceptance of the telescope was intentionally increased. As a consequence, the energy resolution obtained was not good enough to clearly separate the elastic events from the inelastic ones. This choice was based on the fact that we are interested in the inclusive quasielastic events.

Figure 2(a) shows the measured quasielastic excitation functions for the ${ }^{16,18} \mathrm{O}+{ }^{60} \mathrm{Ni}$ systems, and Fig. 2(b) shows the corresponding barrier distributions, which were derived by using the point difference method with an energy interval of $\Delta E_{\mathrm{LAB}}=2.0 \mathrm{MeV}$. A qualitative analysis of Fig. 2 shows the strong influence of the external pair of neutrons in the ${ }^{18} \mathrm{O}$ nucleus on the direct reaction mechanism at backward angles. In particular, Fig. 2(b) shows that the barrier distributions are very different for the two systems. As can be observed directly from the spectrum of the ${ }^{18} \mathrm{O}+{ }^{60} \mathrm{Ni}$ system, several reaction channels are open and they produce the enlargement of the barrier distribution of this system, since each one of these channels has its own "reaction barrier." As the quasielastic barrier distribution is normalized to 1 , the enlargement of the distribution is followed by the reduction of the height of its main peak. By comparing Figs. 2(a) and 2(b), one can observe how the barrier distribution is much more powerful than the excitation function as a method to compare different systems and to put in evidence details of their reaction mechanisms.

\section{RESULTS FOR THE ${ }^{16} \mathrm{O}+{ }^{60} \mathrm{Ni}$ System}

In this section, we compare the experimental results for the ${ }^{16} \mathrm{O}+{ }^{60} \mathrm{Ni}$ system with theoretical predictions from coupled channel calculations (CCCs). The real nucleus-nucleus

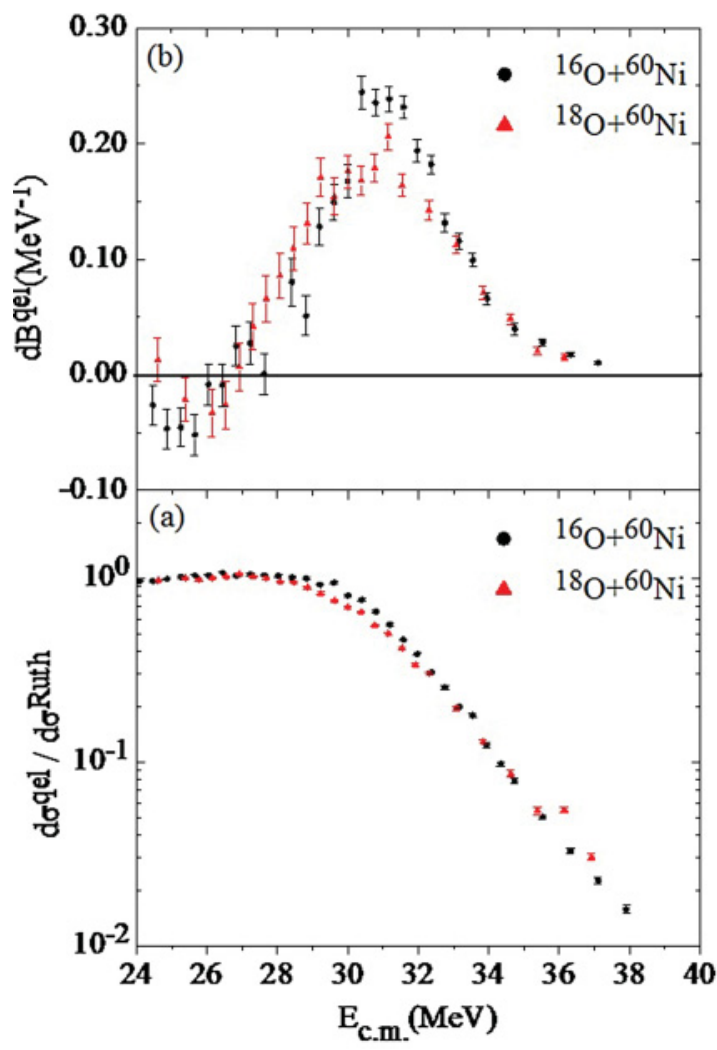

FIG. 2. (Color online) (a) Measured quasielastic excitation functions for the ${ }^{16,18} \mathrm{O}+{ }^{60} \mathrm{Ni}$ systems taken at $\theta_{\mathrm{LAB}}=161^{\circ}$; (b) corresponding quasielastic barrier distributions.

interaction potential used is the double-folding parameter-free São Paulo potential (SPP) [3], [21]. By parameter-free we mean that the potential parameters are obtained from a large and realistic systematic of nuclear densities [3]. In the following, we will compare the data with theoretical predictions without any fit procedure. To avoid the double counting of channels included explicitly in the CCC, we do not include any imaginary potential at the nuclear surface region. The flux that is absorbed from the incident channel and goes to the fusion channel is taken into account by a very short-range imaginary potential of Woods-Saxon form with the following parameters: $W_{i}=80.0 \mathrm{MeV}, r_{i}=0.9 \mathrm{fm}$, and $a_{i}=0.2 \mathrm{fm}$. All CCCs were performed using the code FRESCO [25].

In the calculations, the nuclear interaction between the colliding nuclei was simulated by a double-folding nuclear potential generated by two-parameter Fermi densities with diffuseness parameter values of $0.53 \mathrm{fm}$ (charge) and $0.56 \mathrm{fm}$ (matter) for both projectile and target. These are the values obtained from the systematic of the SPP. All low-lying excited states of the target for which there are experimental values of $B(E \lambda)$ were included in the CCC: the one-phonon states $2_{1}^{+}$and $3_{1}^{-}$, and the two-phonon triplet $2_{2}^{+}, 0^{+}$, and $4^{+}$. For the ${ }^{16} \mathrm{O}$ projectile, only its ground state was considered. The dotted curves in Fig. 3 are the results for the no-coupling calculations for [Fig. 3(a)] the QES excitation function and [Fig. 3(b)] barrier distribution, respectively. The dashed curves are the results when inelastic excitations of the target were included 

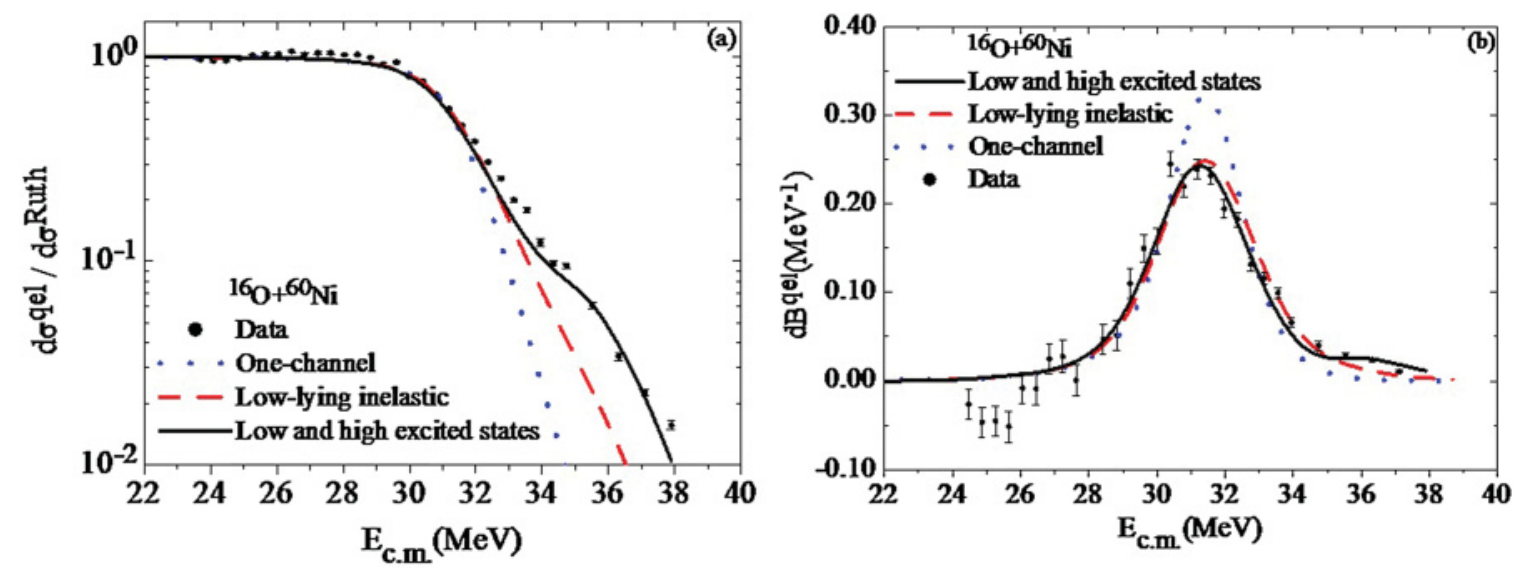

FIG. 3. (Color online) (a) Quasielastic excitation function and (b) quasielastic barrier distribution for the ${ }^{16} \mathrm{O}+{ }^{60} \mathrm{Ni}$ system. The dotted curves are the CCC results without any coupling. The dashed curves are the CCC results when one- and two-phonon excited states of the target are included in the coupling scheme. The solid lines are the calculation including higher excited states of the target (see text for details). These results were obtained assuming the average nuclear matter diffuseness $a=0.56 \mathrm{fm}$ for the ${ }^{16} \mathrm{O}$ and ${ }^{60} \mathrm{Ni}$ nuclei.

in the calculations. The ${ }^{60} \mathrm{Ni}$ excited states included were $1.332 \mathrm{MeV}\left(J^{\pi}=2_{1}^{+}\right)$and the two-phonon triplet $2.158 \mathrm{MeV}$ $\left(J^{\pi}=2_{2}^{+}\right), 2.285 \mathrm{MeV} \quad\left(J^{\pi}=0^{+}\right)$, and $2.506 \mathrm{MeV}$ $\left(J^{\pi}=4^{+}\right)$. The quadrupole deformation parameter used was 0.2070 , taken from Ref. [26]. The coupling of the first $2^{+}$ excited state is the one which produces the most remarkable effect in both cases. Comparing Figs. 3(a) and 3(b) one can observe that, despite a very good agreement between the experimental results and theoretical predictions for the barrier distribution, for the high-energy part of the excitation function a good agreement was not reached by coupling only low-lying target inelastic excitations.

In order to try to improve the agreement between theoretical predictions and data, the $\alpha$-stripping process $\left(Q_{\mathrm{gg}}=\right.$ $3.20 \mathrm{MeV}$ ) was also coupled. However, the results showed that this channel does not significantly influence the QES excitation function and QEBD. Then, the octopole vibration of the projectile was also included in the coupling matrix, but the differences between theoretical and experimental QES excitation functions at energies above the Coulomb barrier still remain. It should be noted that in our CCC there is no imaginary potential at the surface interaction region, and that all reaction flux taken from the elastic channel must be included explicitly in the coupling matrix. Finally, we included higher inelastic excitations of the ${ }^{60} \mathrm{Ni}$ target: $3.124 \mathrm{MeV}\left(J^{\pi}=2^{+}\right)$, $3.269 \mathrm{MeV}\left(J^{\pi}=2^{+}\right)$, and $3.393 \mathrm{MeV}\left(J^{\pi}=2^{+}\right)$. Due to the lack of experimental $B(E \lambda)$ data for these states of the ${ }^{60} \mathrm{Ni}$ nucleus, we used the values of equivalent states of the ${ }^{58} \mathrm{Ni}$ nucleus, for which the experimental values are available. The result of this calculation is represented by the solid lines in Fig. 3, where the agreement between theoretical predictions and the excitation function data was improved [Fig. 3(a)]. This result shows that high states of the target are excited in this system at energies above the Coulomb barrier. On the other hand, Fig. 3(b) shows that the barrier distribution is not influenced very much by these additional channels. This fact confirms that QEBD is not very sensitive to the part of the QES excitation function corresponding to energies well above the barrier. Therefore, we may conclude that the ${ }^{16} \mathrm{O}$ and ${ }^{60} \mathrm{Ni}$ nuclei are well described by the charge and matter diffuseness parameters of $0.53 \mathrm{fm}$ and $0.56 \mathrm{fm}$, respectively. As will be clear in the next section, this result is very important for the method that will be presented for the derivation of nuclear matter diffuseness (NMD) of nuclei.

\section{RESULTS FOR THE ${ }^{18} \mathrm{O}+{ }^{60} \mathrm{Ni}$ SYSTEM AND THE DERIVATION OF THE NUCLEAR MATTER DIFFUSENESS OF THE ${ }^{18} \mathrm{O}$ NUCLEUS}

For the ${ }^{18} \mathrm{O}+{ }^{60} \mathrm{Ni}$ system, a similar procedure to the one used for the ${ }^{16} \mathrm{O}+{ }^{60} \mathrm{Ni}$ was followed. The main difference is that for ${ }^{18} \mathrm{O}$ we have also coupled three transfer channels: one-neutron (1n), two-neutron (2n), and one- $\alpha$ stripping. Table I summarizes the states considered in the following calculations. The same inelastic states for the ${ }^{60} \mathrm{Ni}$ were coupled. For the ${ }^{18} \mathrm{O}$, the first $2^{+}(1.9822 \mathrm{MeV})$ inelastic state was also included in the calculations. The ${ }^{18} \mathrm{O}$ deformation parameter used, 0.355, was taken from Ref. [26]. For the 1n transfer, excited states were considered up to $1 \mathrm{MeV}$, whereas for the $2 \mathrm{n}$ transfer the experimental spectrum showed excited states up to $5 \mathrm{MeV}$ [27]. In the present work, the excited states and their spectroscopic characteristics were taken from Ref. [27]. In the transfer calculations, the prior, full real remnant and one-step cluster transfer with $S=0$ were assumed. The spectroscopic factors for the $1 \mathrm{n}$ transfer were taken as 0.8 , whereas for the $2 n$ transfer, the starting value of 0.6 was obtained from Ref [27]. However, this last value was later slightly changed to 0.5 , in order to achieve a better agreement with the data. For the $\alpha$-stripping reaction, only transfer to the ground state was considered and a spectroscopic factor equal to 1.0 was assumed. Some variations around this value do not significantly change the results.

The nuclear charge and matter diffuseness initially used were the average values of $0.53 \mathrm{fm}$ and $0.56 \mathrm{fm}$, respectively, obtained from the SPP systematic, for both projectile and target. So, once more, the results of the calculations correspond to predictions rather than fit procedure. The results are shown 
TABLE I. The one-neutron and two-neutron tranfer channels coupled in the calculation for the ${ }^{18} \mathrm{O}+{ }^{60} \mathrm{Ni}$ system.

\begin{tabular}{|c|c|c|c|}
\hline${ }^{17} \mathrm{O}$ & ${ }^{61} \mathrm{Ni}$ & ${ }^{16} \mathrm{O}$ & ${ }^{62} \mathrm{Ni}$ \\
\hline $5 / 2^{+}$(g.s.) & $3 / 2^{-}$(g.s.) & $0^{+}$(g.s.) & $0^{+}$(g.s.) \\
\hline $5 / 2^{+}$(g.s.) & $5 / 2^{-}(0.07)$ & $0^{+}$(g.s.) & $2^{+}(1.17)$ \\
\hline $5 / 2^{+}$(g.s.) & $1 / 2^{-}(0.28)$ & $0^{+}$(g.s.) & $0^{+}(2.05)$ \\
\hline $1 / 2^{+}(0.87)$ & $3 / 2^{-}$(g.s.) & $0^{+}$(g.s.) & $2^{+}(2.30)$ \\
\hline $1 / 2^{+}(0.87)$ & $1 / 2^{-}(0.66)$ & $0^{+}$(g.s.) & $4^{+}(2.34)$ \\
\hline \multirow[t]{20}{*}{$1 / 2^{+}(0.87)$} & $5 / 2^{-}(0.91)$ & $0^{+}$(g.s.) & $0^{+}(2.89)$ \\
\hline & & $0^{+}$(g.s.) & $2^{+}(3.06)$ \\
\hline & & $0^{+}$(g.s.) & $2^{+}(3.16)$ \\
\hline & & $0^{+}$(g.s.) & $4^{+}(3.17)$ \\
\hline & & $0^{+}$(g.s.) & $2^{+}(3.26)$ \\
\hline & & $0^{+}$(g.s.) & $2^{+}(3.27)$ \\
\hline & & $0^{+}$(g.s.) & $4^{+}(3.27)$ \\
\hline & & $0^{+}$(g.s.) & $2^{+}(3.37)$ \\
\hline & & $0^{+}$(g.s.) & $2^{+}(3.52)$ \\
\hline & & $0^{+}$(g.s.) & $2^{+}(3.86)$ \\
\hline & & $0^{+}$(g.s.) & $2^{+}(3.97)$ \\
\hline & & $0^{+}$(g.s.) & $4^{+}(3.99)$ \\
\hline & & $0^{+}$(g.s.) & $4^{+}(4.05)$ \\
\hline & & $0^{+}$(g.s.) & $2^{+}(4.06)$ \\
\hline & & $0^{+}$(g.s.) & $0^{+}(4.23)$ \\
\hline & & $0^{+}$(g.s.) & $2^{+}(4.32)$ \\
\hline & & $0^{+}$(g.s.) & $2^{+}(4.42)$ \\
\hline & & $0^{+}$(g.s.) & $2^{+}(5.00)$ \\
\hline & & $0^{+}(6.05)$ & $0^{+}$(g.s.) \\
\hline & & $3^{-}(6.13)$ & $0^{+}$(g.s.) \\
\hline
\end{tabular}

by the dashed curves in Fig. 4(a) for the QES excitation function and Fig. 4(b) for the QEBD, respectively. One can observe a large discrepancy between the theoretical predictions and the experimental values in both figures. No improvement could be reached by including other inelastic states and/or varying values of the transfer spectroscopic factors.

As we have previously shown that the ${ }^{17} \mathrm{O}$ nucleus has a NMD of $a=0.62 \mathrm{fm} \mathrm{[10],} \mathrm{and} \mathrm{there} \mathrm{is} \mathrm{some} \mathrm{experimental}$ evidence [28] that the ${ }^{18} \mathrm{O}$ nucleus also presents larger NMDs ( $a=0.60 \mathrm{fm}$ ), when compared with the value from the SPP systematic $(0.56 \mathrm{fm})$, we used for the ${ }^{18} \mathrm{O}+{ }^{60} \mathrm{Ni}$ system the same method described in Ref. [10]. To obtain the best fit to the experimental quasielastic barrier distribution, CCCs were performed with the same coupling scheme as before, but by using double-folding potentials generated with the NMD of the ${ }^{18} \mathrm{O}$ nucleus as the only free parameter. The charge diffuseness of the ${ }^{18} \mathrm{O}$ nucleus was kept as $0.53 \mathrm{fm}$, because we assumed that the presence of two neutrons outside the closed shells of the ${ }^{16} \mathrm{O}$ core do not disturb its charge distribution too much. On the other hand, in the last section we proved that the ${ }^{60} \mathrm{Ni}$ nucleus has charge- and nuclear-matter diffuseness equal to $0.53 \mathrm{fm}$ and $0.56 \mathrm{fm}$, respectively. The solid curves in Fig. 4 show the good fits obtained when the NMD of the ${ }^{18} \mathrm{O}$ nucleus assumes the value $0.62 \mathrm{fm}$.

In order to test the sensitivity of our method with the NMD parameter, CCCs were performed with double-folding potentials generated with the NMD of the ${ }^{18} \mathrm{O}$ nucleus ranging from $0.55 \mathrm{fm}$ to $0.67 \mathrm{fm}$, in steps of $0.01 \mathrm{fm}$. For each diffuseness value, a reduced $\chi^{2}$ value was obtained from the fit to the barrier distribution, and a curve of reduced $\chi^{2}$ versus diffuseness parameter was obtained. The analysis of this curve gave a very precise result for the NMD parameter of the ${ }^{18} \mathrm{O}$ nucleus: $0.625 \pm 0.005 \mathrm{fm}$. This uncertainty value corresponds to a variation of $\pm 10 \%$ of the minimum reduced $\chi^{2}$. Therefore, we observe that the near-barrier backward quasielastic scattering can be used as a sensitive tool to determine the NMD of the colliding nuclei.

Once we have determined the correct value of the NMD of the ${ }^{18} \mathrm{O}$ projectile, in the following we will discuss the relative importance of different channels in the $\mathrm{CCC}$ for both quasielastic excitation function and barrier distribution. Figure 5 shows the results of the calculations for [Fig. 5(a)] the QES excitation function and [Fig. 5(b)] the QEBD in the following situations. The dotted curves are the results of the calculations when no couplings are considered. The dashed curves are the CCC results when inelastic excitations of the projectile ${ }^{18} \mathrm{O}\left(2^{+}\right)$
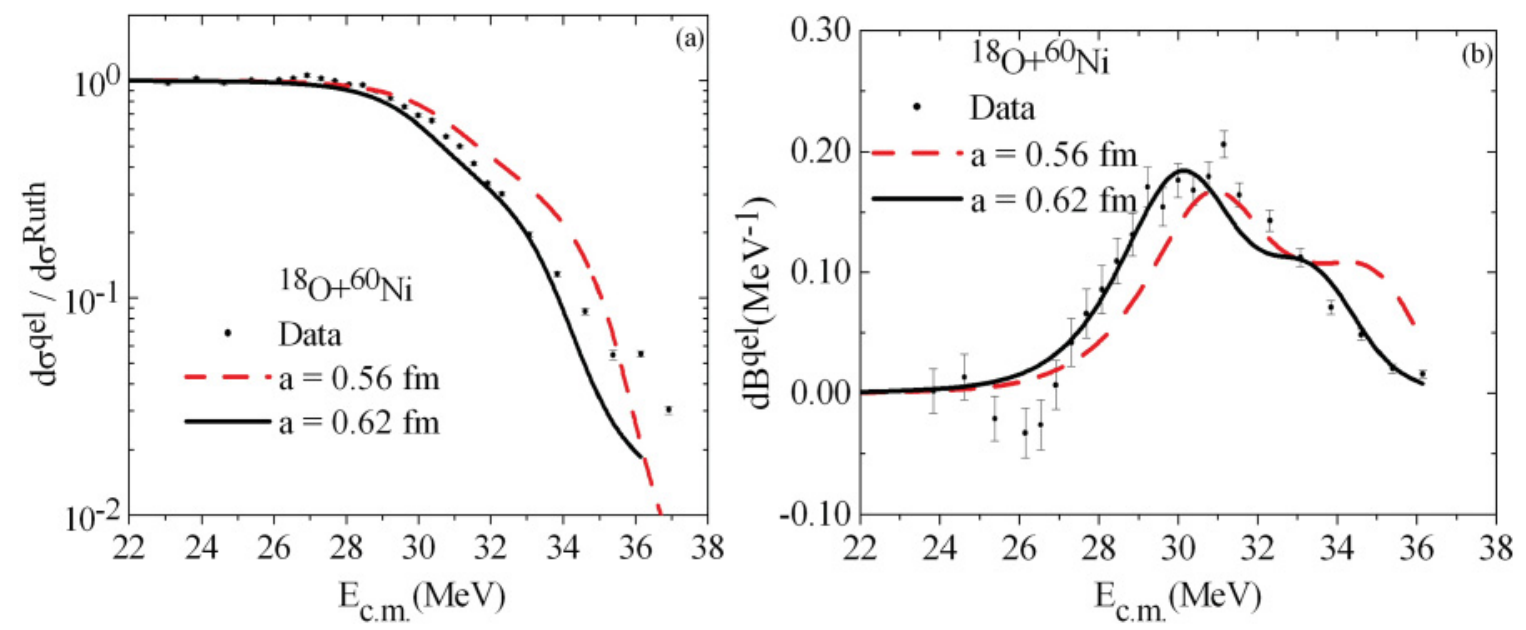

FIG. 4. (Color online) (a) Quasielastic excitation function and (b) quasielastic barrier distribution for the ${ }^{18} \mathrm{O}+{ }^{60} \mathrm{Ni}$ system. The dashed curve is the result for full CCC assuming the NMD value of $0.56 \mathrm{fm}$, for both projectile and target. The solid curve is the result of the same $\mathrm{CCC}$, except for the fact that the projectile NMD was changed to $0.62 \mathrm{fm}$. 

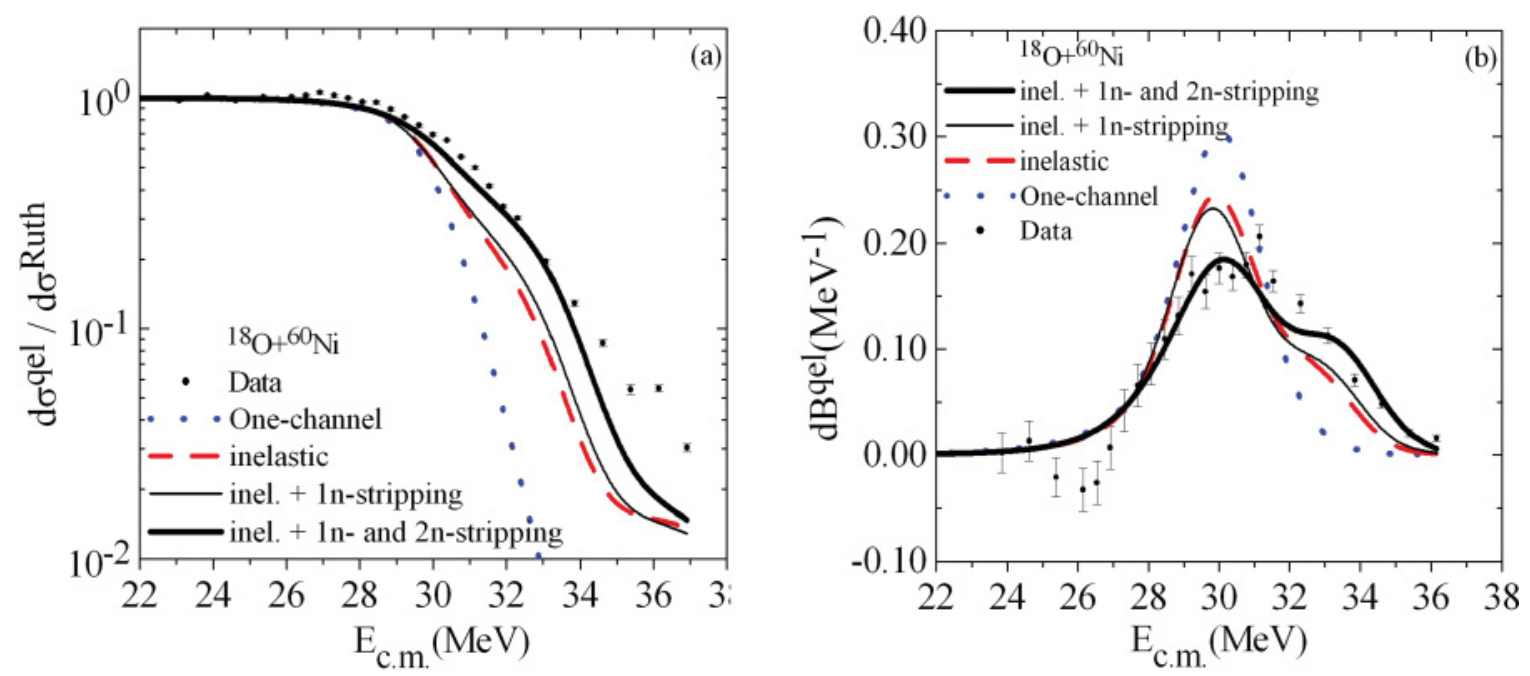

FIG. 5. (Color online) (a) Quasielastic excitation function and (b) quasielastic barrier distribution for the ${ }^{18} \mathrm{O}+{ }^{60} \mathrm{Ni}$ system compared to CCC. The nuclear matter diffuseness of the ${ }^{18} \mathrm{O}$ projectile used was $0.62 \mathrm{fm}$. (See the text for details)

and target ${ }^{60} \mathrm{Ni}\left(2_{1}^{+}, 2_{2}^{+}, 0^{+}, 4^{+}\right)$are included in the coupling scheme. One can observe a remarkable improvement in the agreement with the data, but a good agreement is not yet obtained. Among the inelastic channels coupled, the $2_{1}^{+}$state of ${ }^{60} \mathrm{Ni}$ and $2_{1}^{+}$state of ${ }^{18} \mathrm{O}$ are responsible for most of the coupling effects on the quasielastic scattering. The thin solid lines in Fig. 5 are the results when the one-neutron stripping channels presented in Table I are included in the CCC. The effect of these channels on the QES and QEBD is very small. A good agreement with the experimental results is obtained only when the two-neutron stripping channels of Table I are included in the calculations. This is shown by the thick solid curves in Fig. 5. One can observe that the two-neutron transfer channel plays a significant role in the coupling scheme. Finally, as for the previous system, the inclusion of the one- $\alpha$ stripping channel in the coupling matrix has a negligible effect on the quasielastic scattering.

\section{SUMMARY AND CONCLUSIONS}

We have measured very precise quasielastic excitation functions for the ${ }^{16,18} \mathrm{O}+{ }^{60} \mathrm{Ni}$ systems at near-barrier energies, at the backward angle $\theta_{\mathrm{LAB}}=161^{\circ}$. We then derived the corresponding quasielastic barrier distributions. The data were compared with predictions from coupled channel calculations using the double-folding São Paulo potential as the bare potential. For the ${ }^{16} \mathrm{O}$-induced scattering, good agreement was obtained for the barrier distribution by using the projectile default nuclear matter diffuseness of $0.56 \mathrm{fm}$ obtained from the SPP systematic. The most important channel for the couplings is the first excited state of the target, whereas the positive $Q_{\mathrm{gg}} \alpha$-stripping channel has almost no effect in the coupling scheme. For the ${ }^{18} \mathrm{O}$-induced scattering, no agreement could be obtained when we used the same nuclear matter diffuseness of $0.56 \mathrm{fm}$. Then we performed coupled channel calculations including inelastic and transfer channels, and letting the projectile nuclear matter diffuseness be the only free parameter. The best fit to data was obtained for the value $0.625 \pm 0.005 \mathrm{fm}$. Contrary to the ${ }^{16} \mathrm{O}+{ }^{60} \mathrm{Ni}$ system, for the ${ }^{18} \mathrm{O}+{ }^{60} \mathrm{Ni}$ system, apart from the importance of the first excited states of projectile and target, the two-neutron stripping channel also proved to be very important in the coupling scheme. Finally, we believe that the present results show clearly that precise near-barrier quasielastic scattering can be used not just as a sensitive tool to investigate coupling channel effects but also to derive nuclear matter diffuseness of the colliding nuclei.

\section{ACKNOWLEDGMENTS}

This work was partially supported by Fundação de Amparo à Pesquisa do Estado de São Paulo (FAPESP), Conselho Nacional de Desenvolvimento Científico e Tecnológico (CNPQ), Fundação de Amparo à Pesquisa do Estado do Rio de Janeiro (FAPERJ), and CAPES.
[1] C. J. Horowitz, S. J. Pollock, P. A. Souder, and R. Michaels, Phys. Rev. C 63, 025501 (2001).

[2] B. Frois, J. B. Bellicard, J. M. Cavedon, M. Huet, P. Leconte, P. Ludeau, A. Nakada, P. Z. Hô, and I. Sick, Phys. Rev. Lett. 38, 152 (1977), and references therein.

[3] L. C. Chamon, B. V. Carlson, L. R. Gasques, D. Pereira, C. De Conti, M. A. G. Alvarez, M. S. Hussein, M. A. Cândido Ribeiro,
E. S. Rossi Jr., and C. P. Silva, Phys. Rev. C 66, 014610 (2002), and references therein.

[4] O. A. Capurro, J. O. Fernandez Niello, A. J. Pacheco, and P. R. S. Gomes, Phys. Rev. C 75, 047601 (2007).

[5] D. S. Monteiro, J. M. B. Shorto, J. F. P. Huiza, P. R. S. Gomes, and E. Crema, Phys. Rev. C 76, 027601 (2007). 
[6] D. S. Monteiro and P. R. S. Gomes, Phys. Rev. C 77, 017601 (2008).

[7] M. Evers, M. Dasgupta, D. J. Hinde, L. R. Gasques, M. L. Brown, R. Rafiei, and R. G. Thomas, Phys. Rev. C 78, 034614 (2008).

[8] L. R. Gasques, M. Evers, D. J. Hinde, M. Dasgupta, P. R. S. Gomes, R. M. Anjos, M. L. Brown, M. D. Rodríguez, R. G. Thomas, and K. Hagino, Phys. Rev. C 76, 024612 (2007).

[9] K. Wahiyama, K. Hagino, and M. Dasgupta, Phys. Rev. C 73, 034607 (2006).

[10] J. F. P. Huiza, E. Crema, A. Barioni, D. S. Monteiro, J. M. B. Shorto, R. F. Simoes, and P. R. S. Gomes, Phys. Rev. C 82, 054603 (2010).

[11] N. Rowley, G. R. Satchler, and P. H. Stelson, Phys. Lett. B 524, 25 (1991).

[12] A. T. Kruppa, E. Romain, M. A. Nagarajan, and N. Rowley, Nucl. Phys. A 560, 845 (1993).

[13] H. Timmers et al., Nucl. Phys. A 584, 190 (1995).

[14] H. Timmers et al., Nucl. Phys. A 633, 421 (1998).

[15] R. F. Simões et al., Phys. Lett. B 527, 187 (2002).

[16] M. V. Andres, N. Rowley, and M. A. Nagarajan, Phys. Lett. B 202, 292 (1988)
[17] J. F. P. Huiza, E. Crema, D. S. Monteiro, J. M. B. Shorto, R. F. Simões, N. Added, and P. R. S. Gomes, Phys. Rev. C 75, 064601 (2007).

[18] J. M. B. Shorto, E. Crema, R. F. Simoes, D. S. Monteiro, J. F. P. Huiza, N. Added, and P. R. S. Gomes, Phys. Rev. C 78, 064610 (2008).

[19] J. Lubian, T. Correa, P. R. S. Gomes, and L. F. Canto, Phys. Rev. C 78, 064615 (2008).

[20] G. Pollarolo, Phys. Rev Lett. 100, 252701 (2008).

[21] L. C. Chamon, D. Pereira, M. S. Hussein, M. A. Candido Ribeiro, and D. Galetti, Phys. Rev. Lett. 79, 5218 (1997).

[22] E. Crema, L. C. Chamon, and P. R. S. Gomes, Phys. Rev. C 72, 034610 (2005).

[23] D. S. Monteiro et al., Phys. Rev. C 79, 014601 (2009).

[24] D. R. Otomar et al., Phys. Rev. C 80, 034614 (2009).

[25] I. J. Thompson, Comput. Phys. Commun. 7, 167 (1988).

[26] S. Raman, C. W. Nester Jr., and P. Tikkanen, At. Data Nucl. Data Tables 78, 1 (2001).

[27] E. S. Rossi et al., Nucl. Phys. A 707, 325 (2002).

[28] D. Pereira et al., Nucl. Phys. A 826, 211 (2009). 